\title{
Learning reward frequency over reward probability: A tale of two learning rules
}

\author{
Hilary J. Don ${ }^{1}$, A. Ross Otto ${ }^{2}$, Astin C. Cornwall ${ }^{1}$, Tyler Davis ${ }^{3} \&$ Darrell A. Worthy ${ }^{1}$ \\ ${ }^{1}$ Texas A\&M University \\ ${ }^{2}$ McGill University \\ ${ }^{3}$ Texas Tech University
}

Please address correspondence to:

Hilary J. Don, Ph.D.

Department of Psychological \& Brain Sciences

Texas A\&M University

4235 TAMU 77843-4235

hilary.don@tamu.edu 
LEARNING REWARD FREQUENCY OVER REWARD PROBABILITY

\begin{abstract}
Learning about reward and expected values of choice alternatives is critical for adaptive behavior. Although human choice is affected by the presentation frequency of reward-related alternatives, this is overlooked by some dominant models of value learning. For instance, the delta rule learns average rewards, whereas the decay rule learns cumulative rewards for each option. In a binary-outcome choice task, participants selected between pairs of options that had reward probabilities of .65 (A) versus .35 (B) or .75 (C) versus .25 (D). Crucially, during training there were twice as many $\mathrm{AB}$ trials as $\mathrm{CD}$ trials, therefore option $\mathrm{A}$ was associated with higher cumulative reward, while option $\mathrm{C}$ gave higher average reward. Participants then decided between novel combinations of options (e.g., AC). Participants preferred option A, a result predicted by the Decay model, but not the Delta model. This suggests that expected values are based more on total reward than average reward.
\end{abstract}

Keywords: reinforcement learning, delta rule, decay rule, reward frequency, probability learning 
LEARNING REWARD FREQUENCY OVER REWARD PROBABILITY

\section{Introduction}

How do we take into account the amount of experience we have with choice alternatives when making decisions? For example, imagine you are deciding whether to go to an old restaurant that you have visited frequently, or a relatively new restaurant that you have visited only a few times. Your choice could be based on the average quality of each restaurant, for example, the food, service, and atmosphere. Alternatively, your choice could be based on the cumulative number of positive experiences you have had with each restaurant. Since you have had much more experience with the old restaurant, this may then bias you towards choosing it, even if the average quality of the new restaurant has been higher (cf. Bornstein \& D'Agostino, 1992).

Similarly, previous work suggests that the way people learn an estimate of the expected value of an option in reinforcement-learning tasks may not be as simple as the probability of that option yielding a reward. Estes (1976) manipulated the frequency of choice options, and found that probability judgments were heavily influenced by how frequently each option had been encountered. Accordingly, Estes suggested that people are more likely to translate memories of rewarded events associated with each alternative into probability judgments than represent such probabilities directly (cf. Murty et al., 2016). Intriguingly, this issue has been overlooked by dominant learning models which are used to characterize experience-based decision-making.

Learning rules are a key component of formal models of cognition. They dictate how models acquire, update, and maintain information about the values of choice alternatives, the weights between network connections, and the strengths of memories. The way in which learning rules are formulated can therefore have substantial effects on the types of information a model is able to learn, and thus what assumptions cognitive theories explicitly or implicitly make about the mechanisms underlying cognition. For example, the Delta rule (Rescorla \& Wagner, 
LEARNING REWARD FREQUENCY OVER REWARD PROBABILITY

1972; Widrow \& Hoff, 1960; Williams, 1992) updates expected values by learning about reward probability in such a way that does not necessarily capture option frequency. A different albeit less prevalent learning rule, the Decay rule (Erev \& Roth, 1998; Yechiam \& Busemeyer, 2005; Yechiam \& Ert, 2007), updates expected values based on cumulative instances of reward associated with each option, and thus may be more sensitive to frequency differences.

Despite their central importance to models of choice, there has been little work to systematically compare learning rules in their sensitivities to different types of information. Accordingly, the present study examines humans' differential sensitivity to the average and cumulative reward provided by choice options, and assesses how Delta and Decay learning rules might capture these differences.

\section{Delta versus Decay Rules}

Delta rule models update expected values based on prediction error, that is, the difference between what was expected and what was received in a given instance. Expected values will therefore approximate the average reward associated with each option, and the frequency with which each option is experienced will not affect its value. The Delta rule is one of the most commonly used learning rules across domains, including reward and value learning in decisionmaking, category learning, and associative learning paradigms (e.g. Busemeyer \& Stout, 2002; Daw et al., 2006; Gluck \& Bower, 1988; Jacobs, 1988; Rumelhart \& McClelland, 1986; Sutton \& Barto, 1981; 1998). Beyond its ability to account for learning behavior, the Delta rule has continued to grow in popularity due to its ability to explain aspects of how dopaminergic brain regions encode prediction errors (Schultz \& Dickinson, 2000; McClure, Berns, \& Montague, 2002; Pessiglione et al., 2006; Samanez-Larkin et al., 2014). 
LEARNING REWARD FREQUENCY OVER REWARD PROBABILITY

Rather than updating expected values on the basis of prediction error, the Decay rule learns a cumulative representation of reward associated with each option - in other words, value estimates are dictated by how often (in total) each option has yielded reward in the past. In turn, options that have been encountered more frequently should be associated with more reward overall, and should receive greater value. The decay rule assumes that outcomes are stored directly in memory, but this memory trace decays over time. This decay parameter means that expected values for each option will be updated on every trial. In comparison, the Delta rule only updates information for the chosen option when prediction error occurs.

In summary, the key difference between the Delta and Decay rules is that the Delta rule will learn the average reward provided by each option, while the Decay rule will learn a decaying representation of the cumulative reward associated with each option. Typically, the same options that provide greater rewards on average are also associated with the highest cumulative reward, such that Delta and Decay rules often make similar predictions. However, their predictions will diverge when choice options are presented at different frequencies.

\section{Binary Choice Task}

In this study, we examined Delta and Decay rule model predictions in two-alternative choice tasks in which participants received binary rewards based on fixed probabilities tied to each option. On some trials, participants learned to choose between option A, rewarded $65 \%$ of the time and option B, rewarded $35 \%$ of the time. On other trials, participants learned to choose between option $\mathrm{C}$, rewarded $75 \%$ of the time, and option $\mathrm{D}$, rewarded $25 \%$ of the time. Critically, participants were given 100 AB trials, but only $50 \mathrm{CD}$ trials. This should create a situation where option $\mathrm{A}$ is associated with the most cumulative reward, whereas option $\mathrm{C}$ has provided the most reward on average. Upon test, participants were presented with several 
LEARNING REWARD FREQUENCY OVER REWARD PROBABILITY

different combinations of the choice options. The key comparison occurs on CA trials, as the Delta rule should predict more $\mathrm{C}$ choices (higher average reward), while the Decay rule should predict more A choices (higher cumulative reward).

\section{Study 1 - Model Simulations}

To verify these empirical predictions we simulated the task with each model using a broad range of parameter value combinations sampled evenly across the parameter space.

\section{Method}

\section{Model Formalisms}

For the Delta rule model, if rewards (r) are coded as 1 when a reward is given and 0 when a reward is not given then the expected value (EV) for each $j$ option is computed on each $t$ trial as:

$$
E V_{j}(t+1)=E V_{j}(t)+\alpha \cdot\left(r(t)-E V_{j}(t)\right) \cdot I_{j}
$$

Where $I_{j}$ is simply an indicator value that is set to 1 if option $j$ is selected on trial $t$, and 0 otherwise. Critically, the update function on the delta rule means that expected values are only updated for the chosen selection. If participants choose $\mathrm{A}$ for an $\mathrm{AB}$ pair, they update their information about $\mathrm{A}$, but not $\mathrm{B}$. The portion of Equation 1 in parentheses is known as the prediction error, and it is modulated by the learning rate parameter $(0 \leq \alpha \leq 1)$. Higher values of $\alpha$ indicate greater weight to recent outcomes, while lower values indicate less weight to recent outcomes. When $\alpha=0$ no learning takes place and expected values remain at their starting points, and when $\alpha=1$ expected values are equal to the last outcome received for each option.

The predicted probability that option $j$ will be chosen on trial $t, P\left|C_{j}(t)\right|$ is calculated using a Softmax rule: 
LEARNING REWARD FREQUENCY OVER REWARD PROBABILITY

$$
P\left|C_{j}(t)\right|=\frac{e^{\beta \cdot E V_{j}(t)}}{\sum_{1}^{N(j)} e^{\beta \cdot E V_{j}(t)}}
$$

Where $\beta=3^{c}-1(0 \leq c \leq 5)$, and $c$ is an inverse temperature parameter that determines how consistently the option with the higher expected value is selected (Yechiam \& Ert, 2007). When $c=0$ choices are random, and as $c$ increases the option with the highest expected value is selected most often. Defining $\beta$ in this way allows it to take on a very large range of values $(0-242)$.

The Decay Rule model also tracks expected values, but it does so without utilizing prediction errors. Specifically, on each $t$ trial the EV for each $j$ option is updated according to:

$$
E V_{j}(t+1)=E V_{j}(t) \cdot A+r(t) \cdot I_{j}
$$

As in Equation $1, I_{j}$ is an indicator variable that is set to 1 if option $j$ was selected on trial $t$, and 0 otherwise. $A$ is a decay parameter, and $r(t)$ is the reward given on each trial. The Decay rule model also uses the Softmax rule presented in Equation 2.

\section{Simulations}

We simulated data sets for each combination of $\alpha$ or $A$ and $c$. $\alpha$ or $A$ varied from 0 to 1 in increments of .05 , and $c$ varied from 0 to 5 in increments of .25. For each parameter combination, we performed 1,000 simulations where each model learned the training phase of the task. After the training phase, we then took each model's predicted probability of choosing $\mathrm{C}$ on the critical CA trials (see Equation 2), and these probabilities were averaged across the 1,000 simulations for each parameter combination.

\section{Results \& Discussion}

Figure 1 plots the average probability of selecting option $\mathrm{C}$ on $\mathrm{CA}$ trials for each parameter combination for the Delta (a) and Decay (b) models. Delta model predictions range from .5 to about .75, while Decay model predictions range from about .15 to .55 . Overall, the Delta model 
generally predicts more $\mathrm{C}$ choices, while the Decay model generally predicts more A choices. Thus, this task is useful for evaluating whether human behavior is more in line with Delta or Decay model predictions that expected value is based on average or cumulative reward, respectively. Study 2 therefore tested how well these predictions align with human behavior.

a.

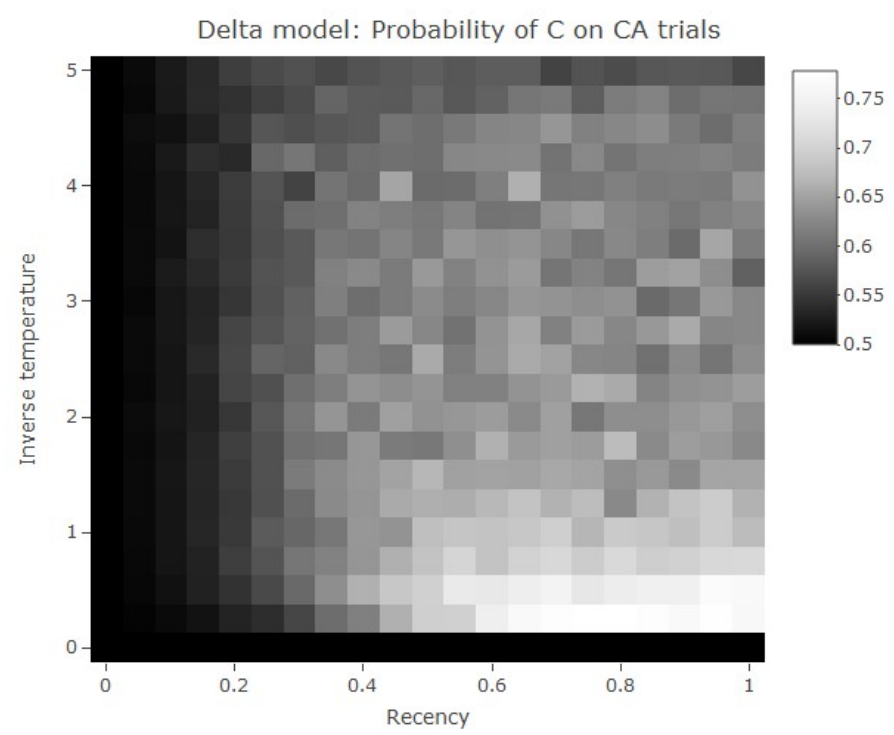

b.

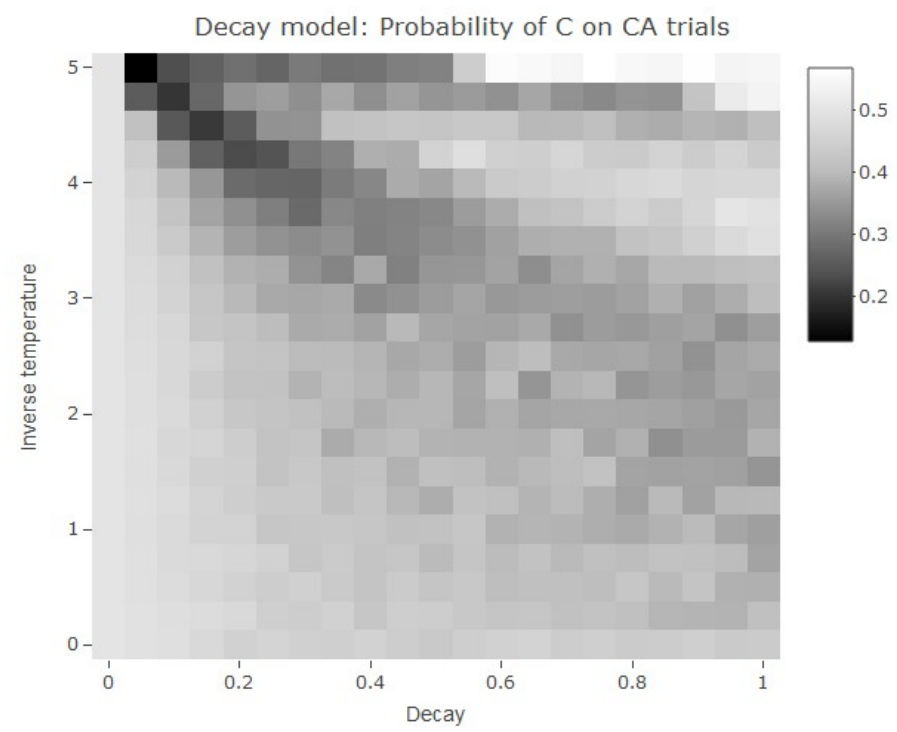

Figure 1. The average probability of selecting $C$ on CA trials across 1,000 simulations for each parameter combination from a) the Delta model and b) the Decay model. 
LEARNING REWARD FREQUENCY OVER REWARD PROBABILITY

\section{Study 2 - Choice Experiments}

To summarize, the Delta rule model assumes that people learn average reward or average reward probabilities, and that the frequency with which each alternative has been encountered should not affect the value of each alternative. The Decay rule assumes that people learn a cumulative representation of reward associated with each option. Options that have been more frequently rewarded should hold higher value.

To test these predictions, we conducted three choice experiments, which were all variants of the same basic task design. The critical components of the task remained consistent across experiments so we present them together for concision. We were most interested in choices made on CA test trials. From the simulations above it is clear that the Delta model predicts more C choices, while the Decay model predicts more A choices. We evaluated choices on these trials and performed fits of each model to the choice data to evaluate which model best characterizes the observed human behavior.

\section{Method}

\section{Participants}

One-hundred thirty-three participants from Texas A\&M University participated in the experiment for partial fulfillment of a course requirement. The Internal Review Board approved the experiment, and participants gave informed consent. Sample sizes for Experiments 1, 2, and 3 were 33, 50, and 50, respectively. Experiment 1 was completed near the end of a term, with the goal of running participants up until that point. The sample size of 50 for the last two experiments was determined semi-arbitrarily, and as a value with enough power to conduct one-sample t-tests for choice proportions on test trials against specific expected choice proportions (detailed below). 
LEARNING REWARD FREQUENCY OVER REWARD PROBABILITY

For the critical comparison of choice proportions on AC trials, with 50 participants we had about .96 power to detect an effect, assuming a moderate effect size of $\mathrm{d}=0.50$.

\section{Materials and Procedure}

Participants performed the experiment on PCs using Matlab software with Psychtoolbox2.54. Participants first completed several questionnaires that are described in the Supplementary Materials. The reward structure was identical to the task described in the introduction. Figure 2a shows a schematic depiction of the tasks. Participants were told that they would make repeated choices on each trial and that they would either receive a reward of one point or zero points on each trial. During the first 150 trials of the task participants made choices between options A versus $\mathrm{B}$, or $\mathrm{C}$ versus $\mathrm{D}$. There were $100 \mathrm{AB}$ trials, and $50 \mathrm{CD}$ trials. Trial types were randomly distributed over the 150 training trials. The reward probabilities associated with options A-D were $\left[\begin{array}{ll}6 & .35 \\ 6 & .25\end{array}\right]$. Upon selecting an option, a random number from 0 to 1 was drawn from a uniform distribution in the background of the computer program. If the number drawn was less than the probability of reward associated with each option then participants received one point, otherwise they received zero points. 
a

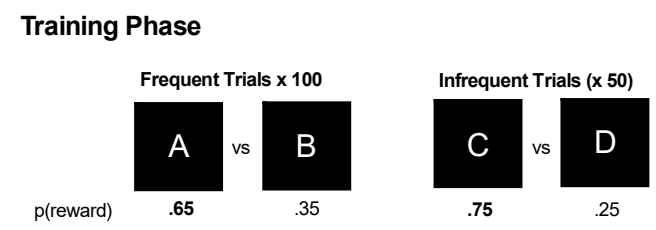

Test Phase

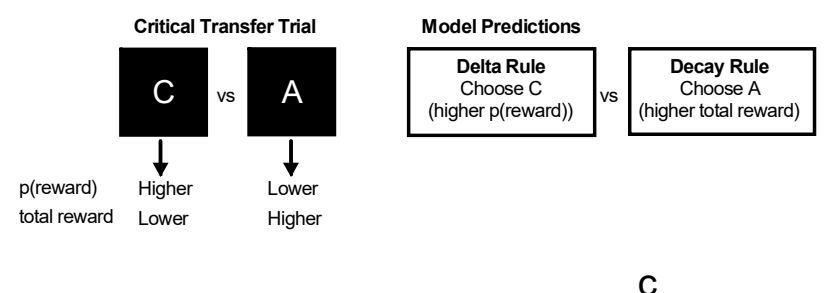

b
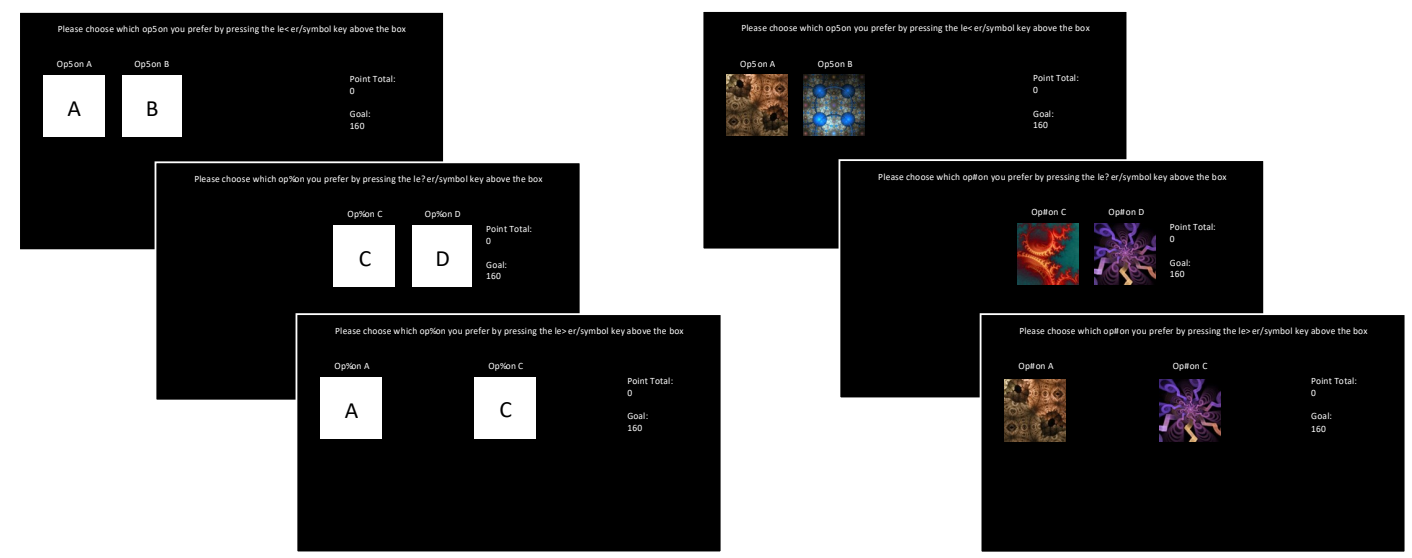

Figure 2. Task details including a) a schematic depiction of the task trial structure and model predictions, b) example screenshots from Experiment 1, and c) example screenshots of Experiment 2 and Experiment 3.

After 150 training trials participants were told that they would now make choices between different pairs of options. They then performed 100 additional trials with pairs $\mathrm{CA}, \mathrm{CB}, \mathrm{AD}$, and BD. There were 25 trials of each type randomly distributed across test trials. In Experiments 1 and 2 participants received feedback in the test phase as well, but in Experiment 3 this phase was performed without feedback. In Experiment 2 and 3 participants also completed a final 50-trial phase where they could select from any of the four options. The results of this phase, which are of no specific interest to the present study, are presented in the Supplementary Material. Participants were not given monetary bonuses in Experiment 1, and only told to earn as many 
LEARNING REWARD FREQUENCY OVER REWARD PROBABILITY

points as possible. In Experiment 2 and 3 participants were offered a monetary bonus of $\$ 0.10$ USD for each point they received in the 100 trial test phase and the final 50-trial four-choice phase.

\section{Data Analysis}

Below we compare the proportion of each objectively optimal choice for each trial type during the learning and test phases of the tasks. For $\mathrm{AB}$ and $\mathrm{CD}$ trials we conduct one-sample ttests against chance, .50 , to determine if, overall, participants learned that $\mathrm{A}$ and $\mathrm{C}$ were the best alternatives within each pair. For the test phase we perform one sample t-tests with the objective reward ratio between the two alternatives as the comparison metric. Thus for CA trials we compare against: $.75 /(.75+.65)=.5357$, and for $\mathrm{CB}, \mathrm{AD}$ and $\mathrm{BD}$ trials against values of $.6818, .7222$, and .5833 , respectively. We also report a comparison for CA trials against a value of .50 , as well as comparisons between each experiment to evaluate whether the results were consistent.

We report the $t$ and $p$-values from each test, as well as Bayes Factors in favor of the alternative hypothesis $\left(\mathrm{BF}_{10}\right)$, in this case that the observed proportion of optimal choice selections differs from the value expected based on the objective reward ratio between the two options. Bayes Factors were computed in JASP (jasp-stats.org) using the default priors. We consider a Bayes Factor of 3 or more to be analogous to a critical threshold, although Bayes Factors can be interpreted continuously as the odds in favor of the alternative hypothesis (Wagenmakers et al., 2018). Bayes Factors less than 1 indicate more support for the null than the alternative hypothesis, and a Bayes Factor less than 1/3 would suggest moderate support for the null hypothesis (analogous to a $\mathrm{BF}_{10}$ of 3 in favor of the alternative hypothesis).

We also fit each model to participants' learning and test data individually. Although both models have the same number of free parameters, we compared the model fits using the Bayesian Information Criterion (BIC; Schwarz, 1978), and examined the degree to which the Decay model 
LEARNING REWARD FREQUENCY OVER REWARD PROBABILITY

fit the data better than the Delta model by computing: $\mathrm{BIC}_{\text {Delta-Decay }}=\mathrm{BIC}_{\text {Delta }}-\mathrm{BIC}_{\text {Decay. }}$ This $\mathrm{BIC}$ difference can then be transformed into a Bayes Factor representing the evidence that the Decay rule is the better model: $\mathrm{BF}_{10 \text {-Decay }}=\exp \left(\mathrm{BIC}_{\text {Delta-Decay }} / 2\right)($ Wagenmakers, 2007$)$.

\section{Data Availability}

Data and experiment and analysis code are available on the Open Science Framework: https://osf.io/t85n6/.

\section{Results}

\section{Training trials}

Across all experiments, there were no differences in optimal choice on AB trials, $F(2,130)=2.69, p=.07, B F_{10}=.68$, or $\mathrm{CD}$ trials, $F(2,130)=3.03, p=.052, B F_{10}=.94$. Overall, participants showed evidence of learning with average $\mathrm{A}$ and $\mathrm{C}$ choices both well above chance, A choices: $M=.663, \mathrm{SD}=.195, t(132)=9.62, p<.001, B F_{10}>1,000 ; \mathrm{C}$ choices: $M=.662, \mathrm{SD}=.209$, $t(132)=8.93, p<.001, B F_{10}>1,000$. There was also no difference in A or $\mathrm{C}$ choices within subjects, $t(132)=0.042, p=.966, B F_{10}=.097$.

\section{CA test trials}

The critical CA test trials determine whether participants prefer the option with higher average reward, or higher reward frequency. Figure 3a shows the average proportion of $\mathrm{C}$ choices on these trials. On average, participants selected $\mathrm{C}$ less often than would be expected from the objective reward ratio, $M=.430, \mathrm{SD}=.289, t(132)=-4.22, p<.001, B F_{10}=347.4$. The median for $\mathrm{C}$ choices was .40. We also conducted a one-sample t-test using a test value of .50. This provided moderate evidence that the proportion of $\mathrm{C}$ choices was lower than would be expected from chance, $t(132)=-2.79, p=.006, B F_{10}=3.96$. Thus, participants selected option A more than would 
LEARNING REWARD FREQUENCY OVER REWARD PROBABILITY

be expected from chance, and much more than would be expected from the objective reward ratio.

Figure $3 \mathrm{~b}$ plots of the distribution of $\mathrm{C}$ choices on $\mathrm{CA}$ trials for each experiment. Visual inspection of these plots suggests some bimodality with some participants clearly preferring option $\mathrm{C}$, but more showing a strong preference toward option A. The plot for Experiment 3 indicates the greatest extent of bimodality; a small subset of participants strongly preferred option $\mathrm{C}$, but most showed a bias toward option A.

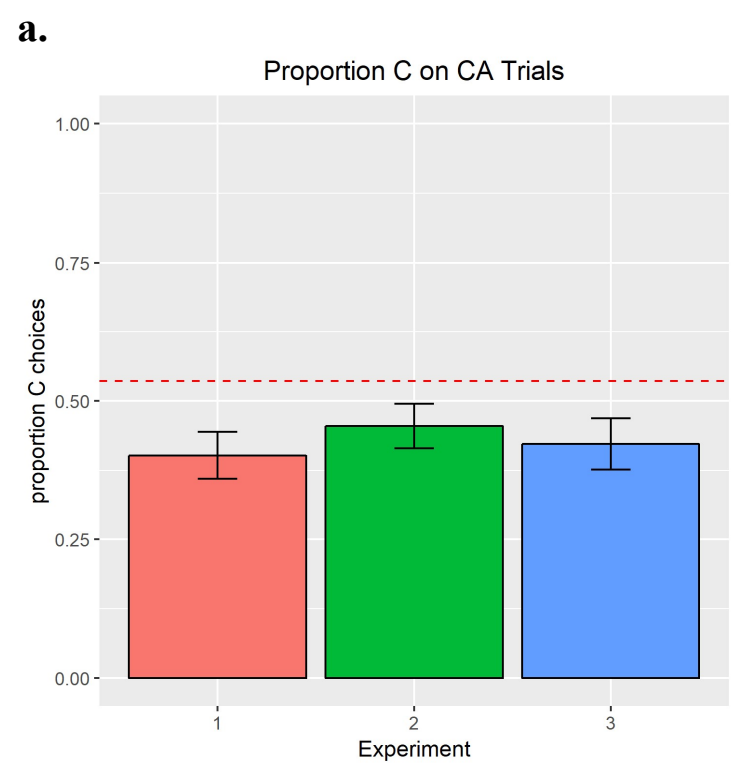

b.

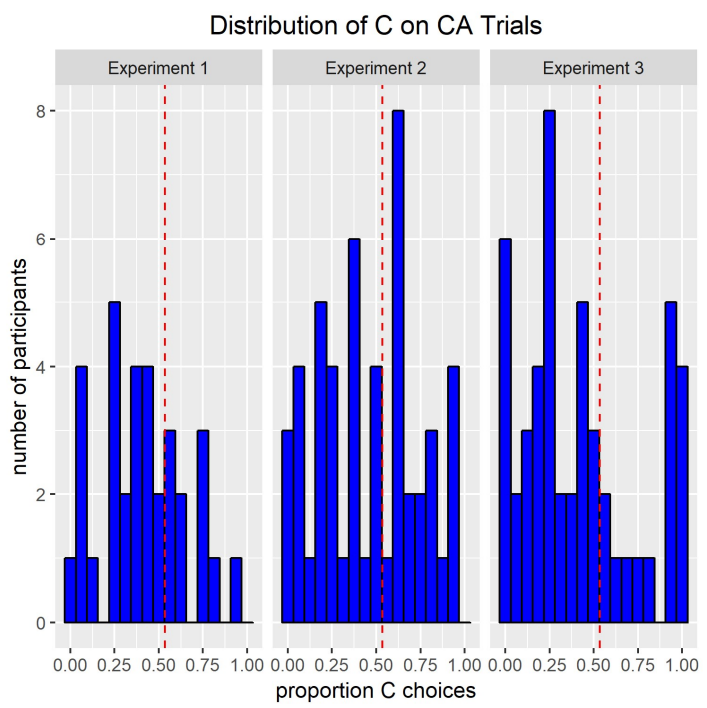

Figure 3. C selections on CA test trials. (a) shows average proportion of $\mathrm{C}$ selections for each experiment. (b) shows the frequency distributions of $\mathrm{C}$ choices on CA test trials where Experiments 1-3 are presented from left to right. Error bars represent standard errors of the mean. 
LEARNING REWARD FREQUENCY OVER REWARD PROBABILITY

\section{Remaining test trials}

On CB test trials, participants chose $\mathrm{C}$ significantly less than the objective reward ratio of $.6818, M=.519, \mathrm{SD}=.318, t(132)=-5.93, p<.001, B F_{10}>1,000$. The median value was .48 . This result also suggests strong effects of presentation frequency. Even though option $\mathrm{C}$ was $40 \%$ more likely to give a reward than option $\mathrm{B}$, many participants did not show a strong preference for option $\mathrm{C}$ on $\mathrm{CB}$ trials. For $\mathrm{AD}$ and $\mathrm{BD}$ trials, participants selected the better alternative in accordance with their objective reward ratios. The proportion of A choices on AD trials did not differ from the reward ratio of $.7222, M=.681, \mathrm{SD}=.275, t(132)=-1.73, p=.086$, $B F_{10}=.407$. Similarly, the proportion of B choices on BD trials did not differ from the reward ratio of $.5833, M=.569, \mathrm{SD}=.281, t(132)=-.598, p=.551, B F_{10}=.115$. Thus, when the most frequently presented items were dominant within a pair, choice probabilities were similar to the objective reward ratios, or probability matching, but when a less frequently presented item was dominant within a pair choice proportions were more consistent with how frequently the item had been presented.

\section{Model Comparison}

To compare the models directly we fit each model individually to each subject's training and test phase data by maximizing the likelihood of each model's next step ahead predictions. The Decay model advantage was taken as $\mathrm{BIC}_{\text {Delta-Decay }}$. Figure 5 plots the average Decay model advantage for each experiment. The average BIC difference was 22.51 which corresponds to a Bayes Factor of over $7.7 \times 10^{4}$ in favor of the Decay model (Wagenmakers, 2007). $72.9 \%$ of participants were best fit by the Decay model; a binomial test suggests this difference is well above $50 \%$ expected by chance, $\mathrm{p}<.001, B F_{10}>1,000$. 
a.

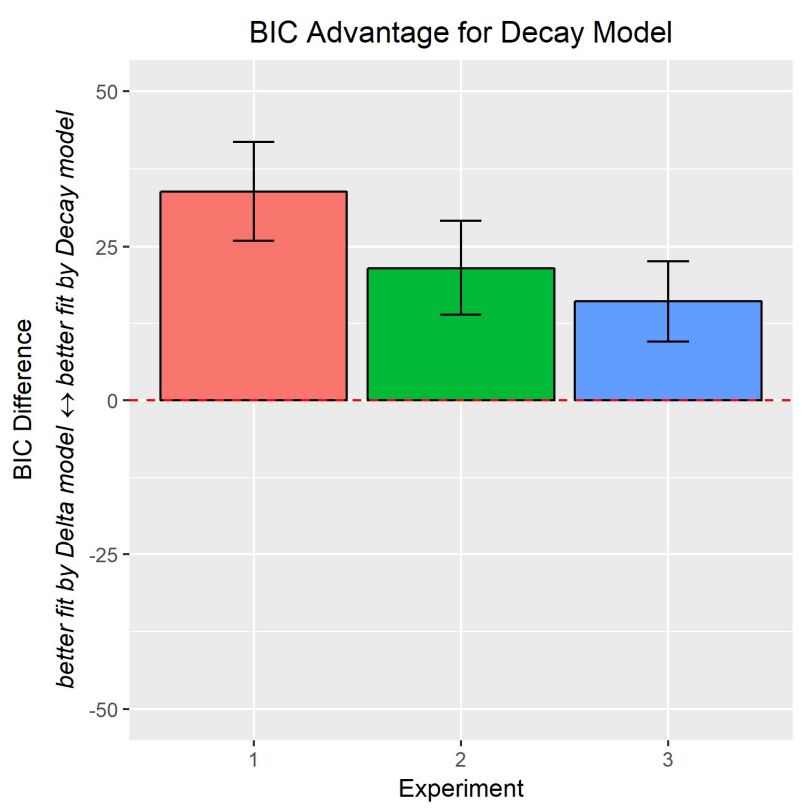

b.

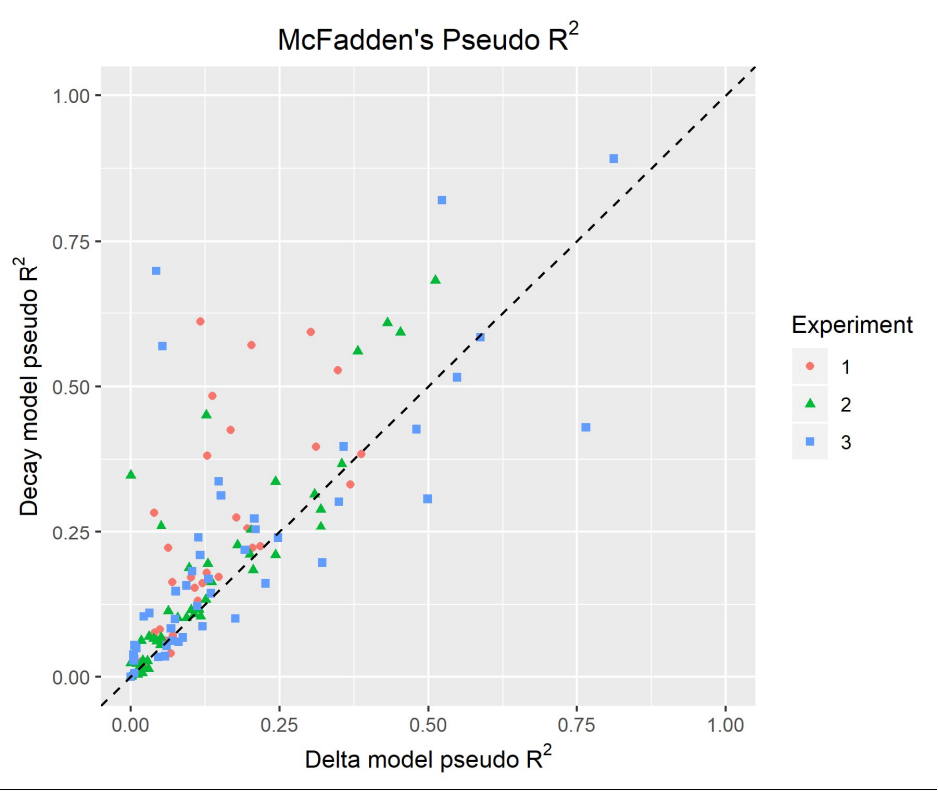

Figure 5. Model comparisons including a) average BIC advantage for the Decay model (BIC Delta-Decay $)$, where a higher score indicates a greater advantage for the decay model, and error bars represent standard errors of the mean, and b) McFadden's pseudo $\mathrm{R}^{2}$ for each participant for the Delta and Decay models, where higher scores indicate greater support for each model over the null model.

Finally, we also compared the Delta and Decay models to a random or null model by computing McFadden's pseudo $\mathrm{R}^{2}$. Both models fit better, overall, than the random model. For the Delta rule model pseudo $\mathrm{R}^{2}$ values for Experiment $1-3$ were $.138, .122$, and .171 . For the 
LEARNING REWARD FREQUENCY OVER REWARD PROBABILITY

Decay rule model pseudo $\mathrm{R}^{2}$ values were $.236, .186$, and .212 . Thus the Decay model explained about $7 \%$ more of the variance in behavior than the Delta model, although a great deal was still left unexplained by either model. To illustrate the model fits on an individual level, pseudo $\mathrm{R}^{2}$ was also calculated for each participant, which are plotted in Figure 5B.

\section{Discussion}

This study tested the influence of reward frequency and reward probability on choice in a reinforcement-learning task, comparing the predictions of two learning rules. We demonstrated that the Delta and Decay rules make divergent predictions about the value of alternative options when those options are presented with different frequency. The Delta rule gives greater value to options with a higher probability of reward, while the Decay rule values options that have yielded more rewards overall. In our experiments, the critical test between the two models was whether participants preferred option $\mathrm{A}$, the more frequently rewarded option, or $\mathrm{C}$, the option with the highest reward probability. Most participants selected option A more than option C on these trials, in support of the Decay model's predictions. The results suggest that participants based their decisions more on how often each option had been rewarded, than on a learned estimate of the probability of receiving reward. This is in line with theories that suggest people do not learn reward probabilities directly, but instead store instances of reward associated with each option in memory and then translate these into choice probabilities that guide their behavior (Estes, 1976; Gonzalez \& Dutt, 2011; Stewart et al., 2006).

The results are compelling as they reveal a clear influence of the amount of experience on choice option value, which is not predicted by one of the most popular learning rules. Delta-based models have also failed to predict other choice effects based on frequency differences. For 
LEARNING REWARD FREQUENCY OVER REWARD PROBABILITY

example, the Rescorla-Wagner model cannot predict the choice preference for a rare outcome in the inverse base-rate effect (Markman, 1989). Delta-based learning is commonly used to model learning of action values from experience in diverse fields such as psychology (Otto \& Love, 2010), computer science and neuroscience (e.g., McClure et al., 2003). Given the prevalence of this formalism — and the assumptions it makes about how value learning unfolds — it is important to validate that the Delta learning model does indeed provide the best account of learning, as operationalized by choice behavior or with neural activity.

Although, our results support the Decay model there is still an extensive body of work that supports predictions from the Delta model (Rangel et al., 2008). A major finding is that prediction errors from the Delta model are correlated activation of the ventral striatum (e.g. Hare et al., 2008; McClure et al., 2003). Additional work can be undertaken to identify whether neural activation in reinforcement-learning tasks is better characterized by Decay rule versus Delta rule prediction errors and expected values. This could potentially be addressed with extant data sets, applying model-based fMRI using each model. In light of the present findings, it is possible that many results previously found to support the Delta rule could also be accommodated by versions of a Decay rule. However, it is important to keep in mind that both models may have aspects that do not align with aspects of neurobiology or cognition (e.g. Steingroever et al., 2014).

Finally, Bayesian versions of the Delta rule model have recently been developed to account for uncertainty in addition to expected value (Gershman, 2015). A Bayesian Decay model may account for more variance in behavior, such as the exploration/exploitation tradeoff, than the simple variant we used here and should be explored in future work. The Decay model may also need to be modified to be more generalizable. A more general version of the Decay model might cumulatively track the number of positive versus negative prediction errors, and would assume 
LEARNING REWARD FREQUENCY OVER REWARD PROBABILITY

that participants make choices based on a recollection of positive versus negative outcomes associated with each option. Such a model would account for the frequency effects we observed here, and also account for the strong neural responses observed for prediction errors (e.g. Hare et al., 2008). Likewise, there are potentially more complicated versions of the Delta model that may allow it to overcome difficulties accounting for frequency information in the present task.

A major point of Estes' (1976) paper is that "probability learning is in a sense a misnomer" or that people do not directly learn reward probabilities (p.51). The current study provides evidence that people place greater value on cumulative instances of reward than on the probability of reward associated with different choice options. We further demonstrate that this is inconsistent with the Delta rule model, which tacitly assumes probability learning. It will be necessary to replicate and extend this work, and further test the key predictions made about learning and behavior by different formal models. 


\section{Notes}

\section{Author Contributions}

D.A. Worthy developed the initial concept of the model simulations and the study. All authors then improved the study concept and developed the study design. D.A. Worthy programmed the simulation code. A.C. Cornwall programmed the experiment code and oversaw data collected under the supervision of D.A. Worthy. H.J. Don, A.C. Cornwall and D.A. Worthy analyzed the data and created the figures. H.J. Don drafted the manuscript under the supervision of D.A. Worthy. A.R. Otto and T. Davis provided critical feedback and significantly revised the manuscript. All authors approved the final version of the manuscript.

\section{Open Practices Statement}

Neither of the studies reported in this article was formally preregistered. The experiment, simulation and code are available on the Open Science Framework: https://osf.io/t85n6/.

\section{Acknowledgements}

This work was supported by NIA grant AG043425 to DAW. We thank research assistants Shannon Yap, Tuyet Linh Huynh, Sumedha Rao, Kirsten Downs, Ashton Wilson, Lilian Garza, Josh Philip, Mikayla Herman, Samantha Rumann, Kaila Powell, Kavyapriya Murali, Kinsey Blackburn, Shannon Pavloske, Marena De-Angelis, Catherine Lee, Melissa Hernandez, Tiffany Dobry, Xavier Jefferson, and lab manager Kaitlyn McCauley for assistance with the data collection. 


\section{References}

Bornstein, R. F., \& D'Agostino, P. R. (1992). Stimulus recognition and the mere exposure effect. Journal of Personality and Social Psychology, 63, 545-552.

Busemeyer, J.R., \& Stout, J.C. (2002). A contribution of cognitive decision model to clinical assessment: Decomposing performance on the Bechara Gambling Task. Psychological Assessment, 14, 253-262.

Daw, N., O’Doherty, J., Dayan, P., Seymour, B., \& Dolan, R. (2006). Cortical substrates for exploratory decisions in humans. Nature, 441, 876-879.

Erev, I., \& Roth, A.E. (1998). Predicting how people play games: Reinforcement learning in experimental games with unique, mixed strategy equilibria. American Economic Review, $88,848-881$.

Estes, W.K. (1976). The cognitive side of probability learning. Psychological Review, 83, 37-64.

Gershman, S.J. (2015). A unifying probabilistic view of associative learning. PLOS Computational Biology, 11, e1004567.

Gluck, M.A., \& Bower, G.H. (1988). From conditioning to category learning: An adaptive network model. Journal of Experimental Psychology: General, 128, 309-331.

Jacobs, R.A. (1988). Increased rates of convergence through learning rate adaptation. Neural Networks, 1, 295-307.

McClure, S.M., Berns, G.S., \& Montague, P.R. (2003). Temporal prediction errors in a passive learning task activate human striatum. Neuron, 38, 339-346.

Murty, V. P., FeldmanHall, O., Hunter, L. E., Phelps, E. A., \& Davachi, L. (2016). Episodic memories predict adaptive value-based decision-making. Journal of Experimental Psychology: General, 145(5), 548-558. 
LEARNING REWARD FREQUENCY OVER REWARD PROBABILITY

Otto, A. R., \& Love, B. C. (2010). You don't want to know what you're missing: When information about forgone rewards impedes dynamic decision making. Judgment and Decision Making, 5(1), 1-10.

Pessiglione, M., Seymour, B., Flandin, G., Dolan, R.J., \& Frith, C.D. (2006). Dopaminedependent prediction errors underpin reward-seeking behavior in humans. Nature, 442, 2006.

Rangel, A., Camerer, C., \& Montague, P. R. (2008). A framework for studying the neurobiology of value-based decision making. Nature Reviews. Neuroscience, 9(7), 545-556.

Rescorla, R.A., \& Wagner, A.R. (1972). A theory of Pavlovian conditioning: Variations in the effectiveness of reinforcement and non-reinforcement. In A.H. Black \& W.F. Prokasy (Eds.) Classical conditioning II: Current research and theory. New York: AppletonCentury-Crofts.

Rumelhart, D.E., McClelland, J.E. \& the PDP Research Group. (1986). Parallel Distributed Processing: Explorations in the Microstructure of Cognition, vols. 1 and 2. Cambridge, MA: MIT Press.

Samanez-Larkin, G. R., Worthy, D. A., Mata, R., McClure, S. M., \& Knutson, B. (2014). Adult age differences in frontostriatal representation of prediction error but not reward outcome. Cognitive, Affective, \& Behavioral Neuroscience, 14, 672-682.

Schultz, W., \& Dickinson, A. (2000). Neuronal coding of prediction errors. Annual Review of Neuroscience, 23, 473-500.

Schwarz, G. (1978). Estimating the dimension of a model. The annals of statistics, 6, 461-464.

Steingroever, H., Wetzels, R., \& Wagenmakers, E.J. (2014). Absolute performance of reinforcement-learning models for the Iowa Gambling Task. Decision, 1, 161-183. 
LEARNING REWARD FREQUENCY OVER REWARD PROBABILITY

Sutton, R.S., \& Barto, A.G. (1998). Reinforcement Learning: An Introduction. Cambridge, MA: MIT.

Wagenmakers, E.J. (2007). A practical solution to the pervasive problems of $p$ values. Psychonomic Bulletin \& Review, 14, 779-804.

Wagenmakers, E.J. et al. (2018). Bayesian inference for psychology Part II: Example applications with JASP. Psychonomic Bulletin and Review, 25, 58-76.

Widrow, B., \& Hoff, M.E. (1960). Adaptive switching circuits. 1960 WESCON Convention Record Part IV, 96-104.

Williams, R.J. (1992). Simple statistical gradient-following algorithms for connectionist reinforcement learning. Machine Learning, 8, 229-256.

Yechiam, E., \& Busemeyer, J.R. (2005). Comparison of basic assumptions embedded in learning models for experience-based decision-making. Psychonomic Bulletin \& Review, 12, 387402.

Yechiam, E. \& Ert, E. (2007). Evaluating the reliance on past choices in adaptive learning models. Journal of Mathematical Psychology, 51, 75-84. 


\section{Supplemental Material}

\section{Measures}

Big Five Inventory (BFI): The BFI is a 44-item list of short, personal, characteristic statements which assesses each participants' personality based on the Big Five factors of Extraversion, Agreeableness, Conscientiousness, Neuroticism, and Openess (John and Srivastava, 1999). For each presented statement, participants will make Likert-style determinations on of how strongly they agree or disagree on a 5-point scale. Each of the five personality factors are independently scored.

Barratt Impulsiveness Scale - 11: The BIS11 consist of 30 questions which describe impulse and non-impulsive behavior (Patton et al., 1995). Participants rated how frequently they partake in the presented behavior on a 4-point scale from "Rarely/Never" to "Almost Always/Always". Participants responses are summed and reported as a total score. Higher scores are indicative of more impulsive behavior.

Narcissistic Personality Inventory - 40 (NPI-40): The NPI-40 includes 40 statements which asks participants to choose 1 of 2 options which is closest to their own feelings about their self (Raskin \& Terry, 1988). For each pair, one statement is considered more narcissistic, and 1 point will be given if chosen. Higher summed points indicate greater narcissistic behavior.

Penn State Worry Questionnaire (PSWQ): The PSWQ is a 16-item list of statements which may characterize a participants' experience with worry independent of depression or anxiety Meyer et al., 1990). Participants rate each statement on a 5-point Likert-scale with 1 being "Not Typical of Me" and 5 being "Very Typical of Me". Responses are summed for a total score of worry. A score range for moderate worry is between 40-59.

Externalizing Spectrum Inventory - Brief (ESI-bf): The ESI-bf is a 160-item list of characteristic statements that participants may relate to, to some degree (Patrick et al., 2013). These statements assess a broad range of problematic behavior related to deficient impulse control. For the present study, we only utilized two of the inventories subscales: the ESI Disinhibition (ESI-Dis; 20 items) and the ESI Substance Abuse (ESI-Sub; 18 items). Higher scores indicate a greater tendency for disinhibited behavior and proneness to abuse drugs or alcohol, respectively.

Center for Epidemiological Studies - Depression (CESD): The CESD is a 20-item questionnaire which asks participants to rate how often, over the course of the past week, they have experienced certain situations that are associated with depressive behavior (Radloff, 1977). The ratings are on a scale of $0-3$ with 0 responses denoting "Rarely/None of the time" and 3 denoting "Most/All of the time". The ratings are summed, higher scores are associated with more depressive behavior.

State-Trait Anxiety Inventory (STAI): The STAI is a 40-item measure used to assess state and trait anxiety levels independent of depressive symptoms (Spielberger et al., 1983). Twenty items each were associated with either state or trait anxiety. Ratings are given on a 4-point Likert-scale 
ranging from "Almost Never" to "Almost Always" with how frequently they associate with the given statement. Higher scores on each subscale, and summed score, indicate greater anxiety.

\section{Experiment 2 \& 3 Final Block Results}

In the final phase of Experiments 2 and 3, all choice options are displayed onscreen simultaneously. For Experiments 2 and 3, the number of times each of the options were chosen over the course of the 50 trials differed significantly: $\mathrm{F}(3,196)=15.849, \mathrm{p}<.001 ; \mathrm{F}(3,196)=.484$, $\mathrm{p}=.004$; respectively.

In Experiment 2, the mean number of times each option was chosen is: $\bar{X}_{\mathrm{A}}=16.60$, $\bar{X}_{\mathrm{B}}=8.40, \bar{X}_{\mathrm{C}}=18.46, \bar{X}_{\mathrm{D}}=6.54$. For the two options of interest, Options A and C, there was not a significant difference in the number of times each was chosen in the final phase, $t(97.77)=-$ $0.7126, p=.4778$. No differences were found between number of $\mathrm{B}$ and $\mathrm{D}$ choices. The option pairings observed in the training trials $(\mathrm{AB}$ and $\mathrm{CD})$ were significantly different: $t(84.689)_{\mathrm{AB}}=3.627, p<.001 ; t(62.342)_{\mathrm{CD}}=6.203, p<.001$.

In Experiment 3, the mean number of times each option was chosen is: $\bar{X}_{\mathrm{A}}=16.69$, $\bar{X}_{\mathrm{B}}=10.30, \bar{X}_{\mathrm{C}}=13.74, \bar{X}_{\mathrm{D}}=9.00$. Options $\mathrm{A}$ and $\mathrm{C}$ still showed no significant differences in the number of times chosen, $t(96.413)=1.207, p=.2303$. As with Experiment 2, no differences were found in the number of B and D choices. Differences between the training trial pairings were again significant: $t(88.13)_{\mathrm{AB}}=2.716, p=.007 ; t(96.104)_{\mathrm{CD}}=2.033, p=.045$.

Between experiments, there were no significant changes in choice frequency from Experiment 2 to Experiment 3. The differences between Option $\mathrm{C}$ choices did approach significance at $t(97.951)=1.874, p=.064$. A graphical depiction of the final choice data for both experiments can be found in Figure S1 below.

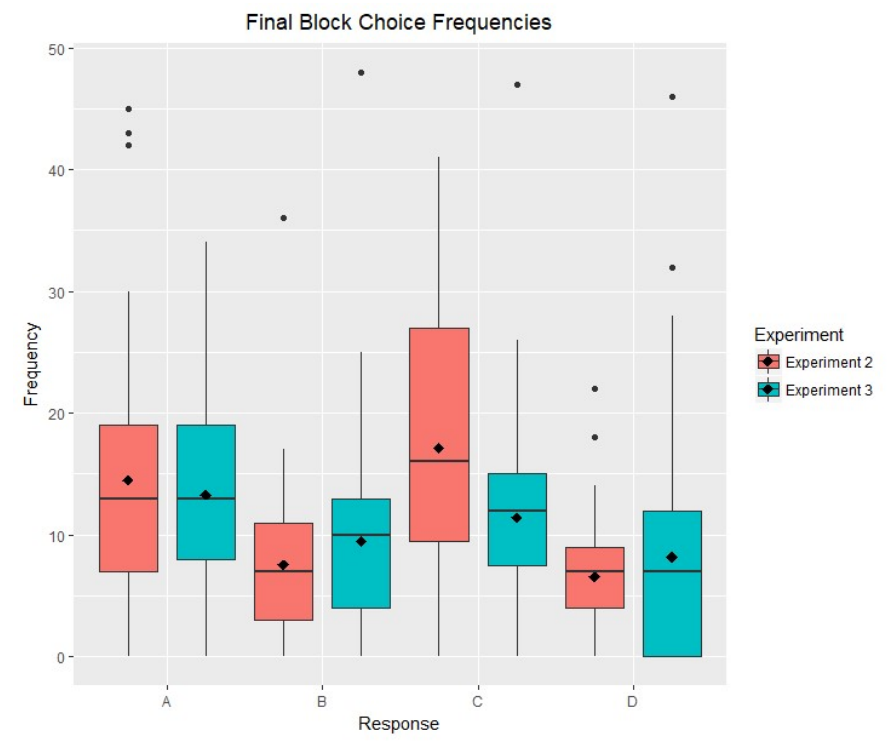

Figure S1: Graphical depiction of final choices made by option letter and by experiment. Means are denoted by a diamond. 


\section{Correlations}

Below we present the correlations between the previously listed personality measures, final phase ABCD choices, and the Delta/Decay BIC Difference by experiment in Figures S2 and S3. For graphical clarity on each plot, the correlation coefficients are rounded to the nearest tenths decimal point. However, highlighted values on the graph represent the non-rounded coefficients greater than 0.3 .

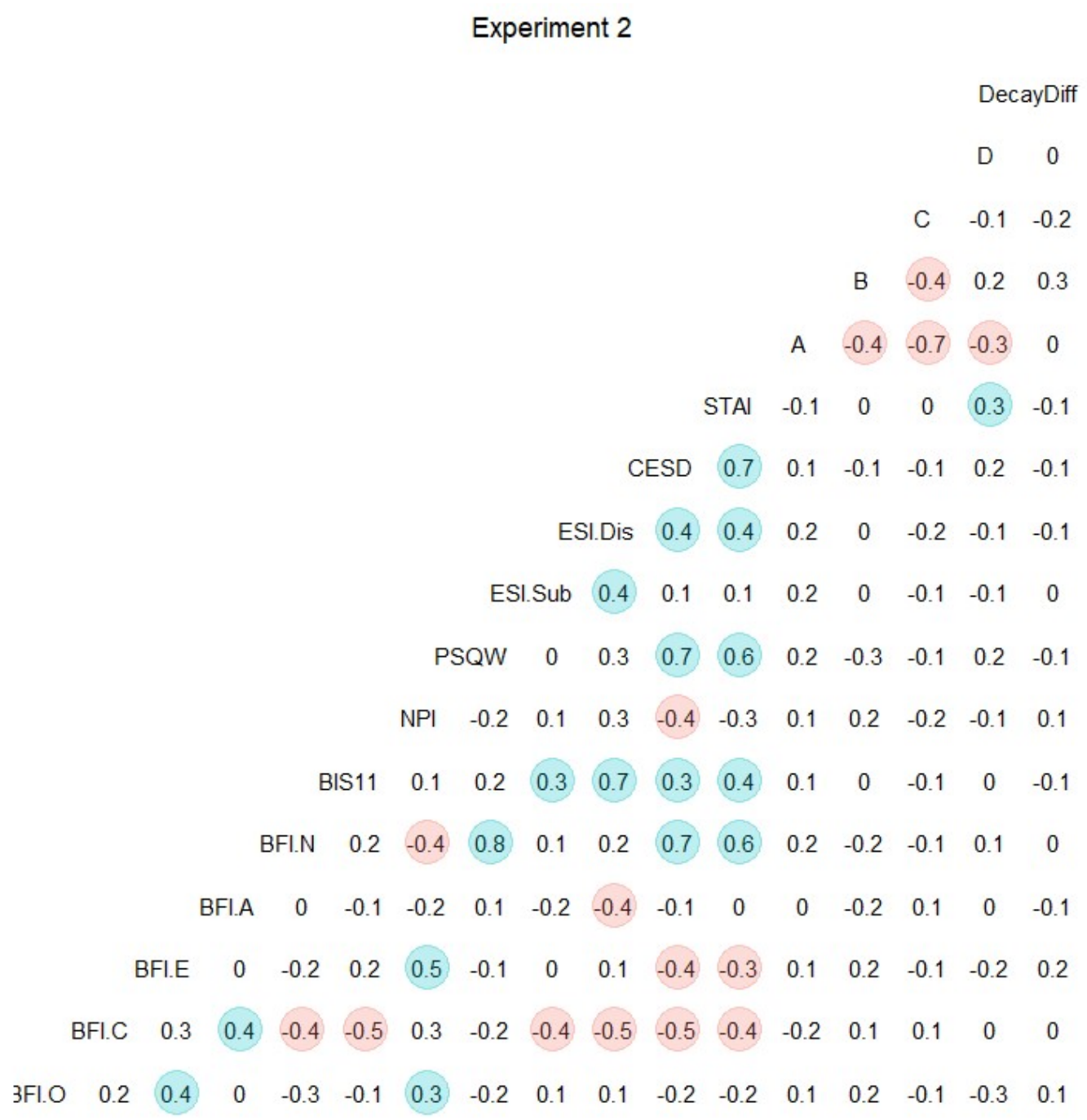

Figure S2: Experiment 2 Correlation Matrix 


\section{Experiment 3}

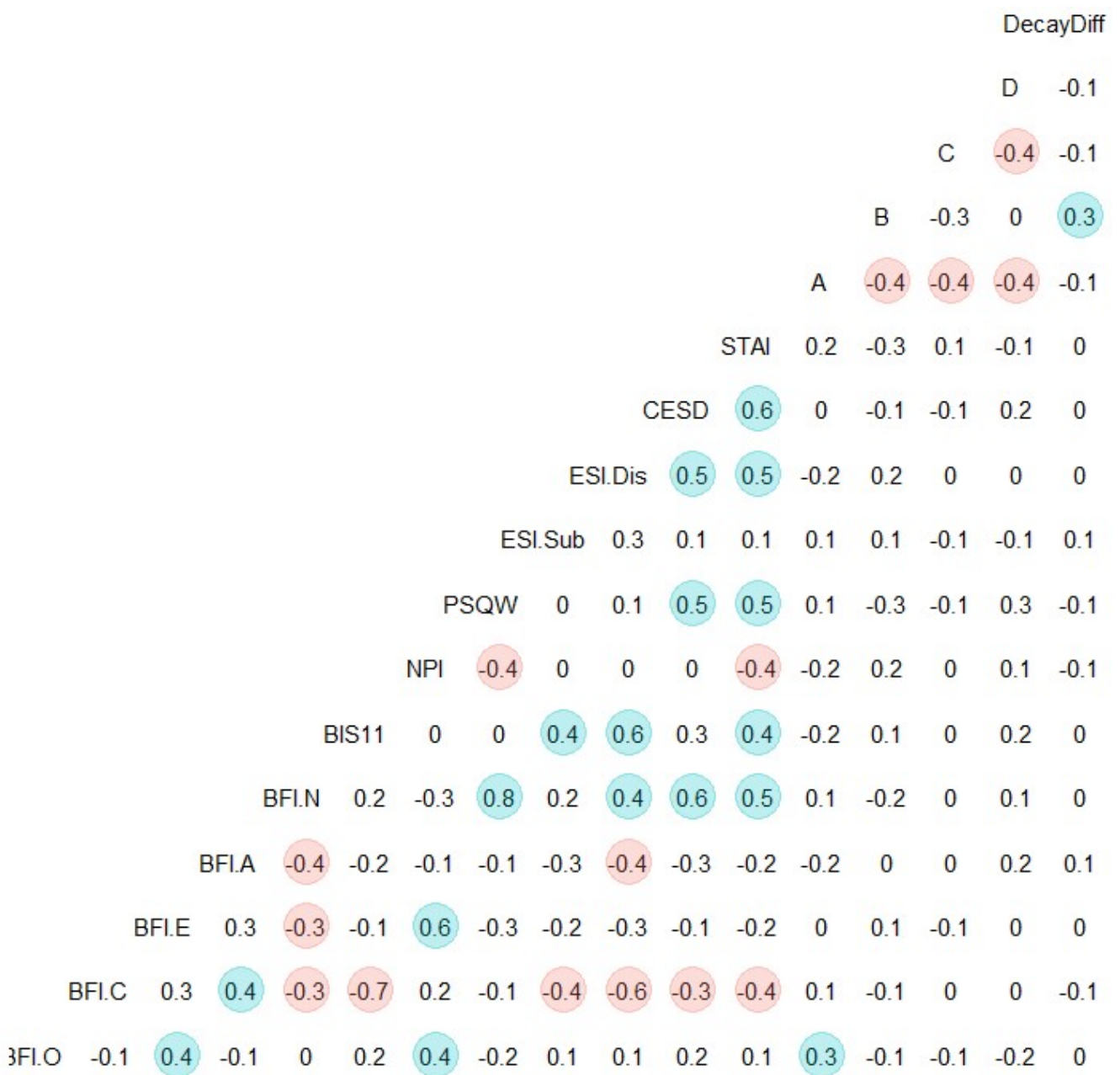

Figure S3: Experiment 3 Correlation Matrix 


\section{References}

John, O. P., \& Srivastava, S. (1999). The Big Five trait taxonomy: History, measurement, and theoretical perspectives. Handbook of personality: Theory and research, 2(1999), 102138.

Meyer TJ, Miller ML, Metzger RL, Borkovec TD (1990). Development and validation of the Penn State Worry Questionnaire. Beh. Research and Therapy, 28, 487-495.

Patrick, C. J., Kramer, M. D., Krueger, R. F., \& Markon, K. E. (2013). Optimizing efficiency of psychopathology assessment through quantitative modeling: Development of a brief form of the Externalizing Spectrum Inventory. Psychological assessment, 25(4), 1332.

Patton, J. H., Stanford, M. S., \& Barratt, E. S. (1995). Factor structure of the Barratt impulsiveness scale. Journal of clinical psychology, 51(6), 768-774.

Radloff, L. S. (1977). The CES-D scale: A self report depression scale for research in the general population. Applied Psychological Measurements, 1, 385-401.

Raskin, R., \& Terry, H. (1988). A principal-components analysis of the Narcissistic Personality Inventory and further evidence of its construct validity. Journal of personality and social psychology, 54(5), 890 .

Spielberger, C. D., Gorsuch, R. L., Lushene, R., Vagg, P. R., \& Jacobs, G. A. (1983). Manual for the State-Trait Anxiety Inventory. Palo Alto, CA: Consulting Psychologists Press. 